\title{
El desarrollo infantil en niños de zonas rurales a partir de la línea de base del Programa Nacional Cuna Más, $2014^{12}$
}

\author{
Child development in rural areas children as from the baseline study of the National \\ Program Cuna Más, 2014
}

David Tarazona C. ${ }^{a C}$, Miguel Campos S. ${ }^{\text {ab}, ~ M i g u e l ~ U g a r e l l i ~ Z . ~}{ }^{\text {ad }}$, José E. Velásquez H. ${ }^{A B}$, Fernando Llanos Z. ${ }^{\text {ab }}$ RECIBIDO 07/03/2016, ACEPTADO 22/05/2016

\footnotetext{
A Ministerio de Desarrollo e Inclusión Social, Dirección General de Seguimiento y Evaluación. Lima-Perú

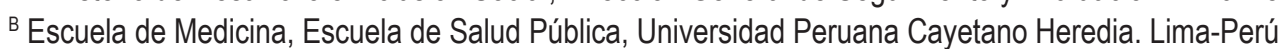

${ }^{c}$ Facultad de Psicología, Universidad Nacional Mayor de San Marcos. Lima-Perú

D Departamento Académico de Economía. Facultad de Ciencias Sociales. Pontificia Universidad Católica del Perú. Lima-Perú
}

\section{RESUMEN}

Objetivos. Conocer los niveles de desarrollo infantil y explorar sus relaciones con el nivel socioeconómico y la educación de la madre. Materiales y métodos. Estudio cuantitativo realizado entre marzo y agosto de 2013, que incluyó información sobre 5859 niños de 1 a menos de 24 meses al momento de la entrevista y sus 5723 madres, provenientes de 5620 hogares en zonas rurales. Se aplicó el Age and Stages Questionnaire ASQ-3 adaptado y una encuesta sociodemográfica. Resultados. Los puntajes de las escalas de comunicación y motora fina mostraron que a medida que la edad es mayor, la brecha en los resultados de las escalas aumenta. En general, los niños del cuartil menos pobre en la muestra obtienen puntajes mayores que los niños del cuartil más pobre para las cinco dimensiones evaluadas. Por otro lado, en las cinco escalas y en el puntaje total se registran diferencias entre los puntajes alcanzados por los niños con madres más y menos educadas. Conclusiones. El estudio ha permitido establecer que existen diferencias en el puntaje obtenido en la prueba ASQ-3 según las variables edad, educación de la madre y niveles de riqueza.

Palabras clave: Desarrollo infantil temprano, ASQ-3, infancia, rural

\begin{abstract}
Objectives. To know the levels of child development and explore its relationship with socioeconomic status and maternal education. Methods. Quantitative - exploratory study done between March and August 2013. The study include information about 5859 children
\end{abstract}

1 El presente artículo ha sido posible gracias a las actividades realizadas en el marco de la Evaluación de Impacto del Programa Cuna Más. En este sentido, los autores desean expresar su agradecimiento al colectivo de instituciones involucradas en este proceso: Banco Interamericano de Desarrollo (BID), Ministerio de Economía y Finanzas (MEF), Instituto Nacional de Estadística e Informática (INEI), Ministerio de Desarrollo e Inclusión Social (MIDIS) y Programa Nacional Cuna Más.

2 Los autores desean agradecer los gentiles comentarios de Luis Baiocchi Paredes, Marco Gonzales Noriega y María Teresa Moreno Zavaleta, los cuales permitieron enriquecer este artículo. 
of 1- 24 months at the interview and their 5723 mothers (or primary caregiver), from 5620 rural homes. We applied an adapted version of Age and Stages Questionnaire ASQ-3 and a socio-demographic questionnaire. Results. Scores in the scales of communication and fine motor showed that, as age is higher, the gap in the results of the scales increases. In general, children less poor quartile in the sample obtain higher scores than children from the poorest to the five dimensions evaluated quartile. On the other hand, there are differences in the five scales and the total score between the scores achieved by children with mothers more and less educated. Conclusions. The study has established that there are differences in the scores obtained in the ASQ-3 based in variables as age, maternal education and wealth level.

Keywords: Early Childhood Development, ASQ-3, Childhood, Rural

\section{INTRODUCCIÓN}

Los resultados de la evaluación censal 2014 del Ministerio de Educación (Minedu) reflejan mejoras en niños de segundo grado de primaria en sus niveles de compresión lectora y matemática; sin embargo, aún $56.5 \%$ de alumnos no comprende lo que lee y el $74 \%$ no tiene las habilidades matemáticas para su grado, y se mantienen amplias brechas según el área geográfica de ubicación de las escuelas y el tipo de gestión de estas (Minedu, 2014). El desarrollo de habilidades durante la niñez y su influencia en la vida futura de un individuo es un tema de investigación clave para el desarrollo de un país.

Grantham-McGregor y otros han reportado evidencia proveniente de estudios longitudinales en Guatemala y Sudáfrica, en que las mediciones de habilidades cognitivas en el nivel preescolar o el primer grado de educación básica correlacionan con el rendimiento académico en educación secundaria, lo cual es complementado con estudios en Filipinas, Brasil y Jamaica, en donde el rendimiento escolar de adolescentes fue predicho por medidas del desarrollo cognitivo a partir de modelos de regresión múltiple controlados por edad, sexo, educación materna y la riqueza, por lo cual se desprende que el desarrollo cognitivo alcanzado en edades tempranas es un predictor del logro académico escolar futuro (Grantham-McGregor y cols, 2007). Otros estudios han reportado que el bajo logro académico en la etapa escolar fue predicho por la malnutrición en los primeros años de vida (Duckworth, 2006; Barnett, 1995). Por otro lado, algunos estudios han hallado que los niños que han presentado rezagos en el desarrollo físico, cognitivo o socioemocional antes de los seis años tuvieron a futuro bajo rendimiento en la escuela y percibieron menores ingresos en la adultez (Schady, 2006; Leseman, 2002; Heckman, 2000).

Considerando la evidencia científica internacional, en el Perú se ha formulado una política pública para promover el desarrollo infantil temprano (DIT) ${ }^{3}$. El Programa Nacional Cuna Más (PNCM) fue creado el 23 de marzo de 2012 a partir del Programa Nacional Wawa Wasi, existente desde principios de la década de 1990. El objetivo del PNCM es "mejorar el nivel de desarrollo físico, cognitivo, comunicacional y socioemocional de niños/as menores de 36 meses de edad

3 PERÚ: Lineamientos para la Gestión Articulada Intersectorial e Intergubernamental orientada a Promover el Desarrollo Infantil Temprano "Primero la Infancia". Resolución Suprema N 413-2013-PCM. 
residentes en zonas en situación de pobreza”. Los resultados intermedios que persigue el programa son "mejorar los conocimientos y prácticas de las madres (padres y/o cuidadores principales) en relación con el cuidado y aprendizaje de sus niños menores de 36 meses y fortalecer el vínculo afectivo entre la madre y su niño para mejorar la calidad de sus interacciones". Para lograr lo propuesto, pone en práctica dos servicios: el Servicio de Acompañamiento a Familias (SAF) y el Servicio de Cuidado Diurno (SCD).

El SAF se desarrolla en zonas rurales y se organiza en torno a visitas domiciliarias. El personal del programa visita a las madres en su hogar y les brinda asesoría personalizada para fortalecer sus capacidades para estimular el desarrollo de sus hijos/as. Esta actividad es llevada a cabo por mujeres de la comunidad, debidamente capacitadas y supervisadas.

El presente artículo muestra resultados del estudio de línea de base del ensayo de evaluación de impacto del SAF del PNCM. El artículo explora si los niveles de desarrollo infantil -medido en forma general y también en las dimensiones de comunicación, motora fina, motora gruesa, resolución de problemas y socioindividual- presentan alguna asociación con la edad, el nivel socioeconómico de las familias y, por otro lado, con el nivel educativo de la madre.

\section{MATERIALES Y MÉTODOS}

\section{Tipo y diseño}

Estudio de tipo cuantitativo-exploratorio transversal, basado en encuestas de hogares realizadas para la línea de base del ensayo aleatorio de evaluación de impacto del Servicio de Acompañamiento a Familias (SAF) del Programa Nacional Cuna Más.

\section{Población y muestra}

Para determinar los distritos y centros poblados a incluir en el estudio, se siguieron los siguientes pasos:

i) Se seleccionaron centros poblados que no contaban con un mínimo de 21 niños por varias razones: a) la implementación de la intervención, en particular, las sesiones grupales que requieren como mínimo de 10 a 12 niños, sería compleja y costosa en centros poblados muy pequeños, con una alta probabilidad de inasistencia de las madres, b) el recojo de datos de la encuesta sería más costoso en centros poblados tan pequeños, y c) en centros poblados tan pequeños habría más incertidumbre sobre el número actual de niños en el rango de edad de la muestra, teniendo en cuenta que la información utilizada proviene del CPV del año $2007^{4}$.

4 De hecho, el empadronamiento previo realizado en campo para la selección de la muestra de niños confirmó una menor 
Dado que se requería 180 distritos para la evaluación, el punto de corte se estableció en un mínimo de 21 niños por centro poblado, con lo cual se tuvo 195 distritos que cumplen esta condición.

ii) Se ordenaron los 195 distritos según su tasa de pobreza y se armaron 65 tríos de distritos con similar nivel de pobreza: los tres distritos más pobres, los tres siguientes más pobres, y así consecutivamente. La agrupación en tríos se debe a que se tienen dos grupos de tratamiento (T1 y T2) y un grupo de control (C).

iii) Se eligieron aleatoriamente 60 tríos. En cada trío, se asignó aleatoriamente un distrito para cada grupo: T1, T2 y C.

iv) En cada uno de estos 180 distritos, se seleccionó a los dos centros poblados con la mayor cantidad de niños entre 0 y 24 meses, con lo cual se tuvo una muestra de 360 centros poblados. Además, se seleccionó al tercer centro poblado con más niños como reemplazo, en caso de que hubiese menos de 5 niños en los dos primeros.

v) Para definir la muestra de niños y madres, se realizó un barrido censal de los centros poblados de la muestra para identificar a todos los hogares con niños menores de 24 meses. Una vez obtenido este padrón, se seleccionaron aleatoriamente 17 hogares para la encuesta. En aquellos centros poblados con 17 niños o menos se encuestó a todos.

Dada la capacidad de expansión inicial del programa, se trabajó con una muestra de 180 distritos, de los cuales 120 deberían contar con el servicio desde inicios de 2013 y 60 podrían incorporarse luego del levantamiento de la línea de seguimiento.

\section{Instrumentos}

Para la medición de desarrollo de los niños menores de 24 meses se usó el Ages and Stages Questionnaires 3 (ASQ-3), una prueba de tamizaje cuyo objetivo es identificar a los niños en riesgo de presentar algún retraso en el desarrollo; el ASQ-3 recoge información sobre cinco dimensiones: comunicación, motora gruesa, motora fina, resolución de problemas y socioindividual (Squires, 2009). La prueba fue aplicada en español y en quechua (traducida de la versión en castellano por un aplicador bilingüe) $)^{5}$.

Con respecto a la confiabilidad del instrumento, se realizó una adecuación contextual del ASQ-3 para facilitar su aplicación en zonas rurales andinas y amazónicas. También se realizó una prueba de confiabilidad test-retest aplicando el ASQ-3 en dos momentos -con 14 días de intermedio- a una muestra de 26 niños. Los resultados arrojaron un coeficiente de correlación de 0.91 para el puntaje total (ítems

cantidad de niños que la reportada por el CPV.

5 Según el último Censo de Población y Vivienda (INEI, 2007), el 32\% de los centros poblados de la muestra tiene población predominantemente quechuahablante. 
regulares más ítems adicionales) y coeficientes por escala de 0.85 (comunicación), 0.83 (motora gruesa), 0.81 (motora fina), 0.80 (resolución de problemas) y 0.83 (socioindividual).

Con respecto a su validez, se han revisado dos estudios, el primero en una muestra de 40 niños de 24 meses cuyas madres participaron en el New York State Angler Cohort Prospective Pregnancy Study (NYSAC PPS) que se desarrolló entre 1998 y 2002 (Gollenberg y cols, 2009), las correlaciones entre la Escala de Psicomotricidad del Bayley Scales of Infant Development III (BSID-III) y la escala motora gruesa del ASQ fueron de $\mathrm{R}=0.46(\mathrm{P}<0.01)$ y las escalas de comunicación y personal social del ASQ con la escala mental del BSID III, de $\mathrm{R}=0.52$ ( $\mathrm{P}<$ $0.001)$ y $\mathrm{R}=0.45$ ( $\mathrm{P}<0.01)$, respectivamente; mientras que no se encontraron correlaciones significativas entre el BSID-III y las escalas motora fina y resolución de problemas del ASQ; adicionalmente se estimó en el ASQ una sensibilidad de $100 \%$ para el punto de corte de retraso severo y de $39 \%$ para retraso leve o grave y una especificidad de $87 \%$ para el punto de corte de retraso severo y $93 \%$ para retraso leve o grave a los 24 meses. El otro estudio revisado se realizó en una muestra de 334 niños del norte de Ontario, en Canadá, con edades entre los 12 y 60 meses, evaluados entre diciembre de 2007 y septiembre de 2008, reportándose que el ASQ alcanzó valores importantes de sensibilidad (82\%) y especificidad (78\%) para ser utilizado en diversos grupos de edad frente a una prueba similar, el Parents' Evaluation of Developmental Status (PEDS), que alcanzó respectivamente valores de $74 \%$ y $64 \%$ (Limbos \& Joyce, 2011).

Se incorporaron tres ítems adicionales en cada escala con la intención de ampliar la capacidad de la prueba de medir el nivel de desarrollo en el extremo superior de la distribución, y, por ende, obtener una puntuación más cercana a la distribución real de desarrollo entre los niños de la muestra, así como más sensible a los cambios marginales que se esperarían como resultado de la intervención del PNCM. En otras palabras, para cada una de las escalas se agregaron los 3 ítems del módulo siguiente no coincidentes con los ítems en el módulo que se está modificando. Así, los ítems que se agregan difieren por escala y por módulo. Estos ítems adicionales se aplicaron solo si el niño fue capaz de realizar los primeros 6 ítems de alguna escala.

\section{Recolección de datos}

El trabajo de campo estuvo a cargo de personal del Instituto Nacional de Estadística e Informática (INEI). Se llevaron a cabo dos pruebas piloto para evaluar el diseño y estructura de la encuesta, el fraseo de las preguntas, la logística del operativo y establecer el tiempo promedio de aplicación (que se estimó en 1 h 25 min). Previo al operativo de campo, se realizó la capacitación de encuestadores en la prueba ASQ-3, durante 19 días, mediante sesiones teóricas y prácticas (entrevistas en aula con madres y niños). Se contó con 77 aplicadores, 45 supervisores locales y 12 coordinadores departamentales. 


\section{Análisis estadístico}

El procesamiento y posterior análisis fueron realizados con el paquete estadístico STATA 13. Los hogares fueron clasificados según su nivel de riqueza mediante un índice construido ad-hoc, en el que se consideraron las variables referentes a la disponibilidad de activos y servicios y los materiales de la vivienda. Sin embargo, no fueron incluidas en el índice todas las variables disponibles. El primer criterio para la inclusión de las variables fue su variabilidad, descartándose los bienes, servicios o materiales que estaban presentes en menos del $10 \%$ de los hogares de la muestra. El segundo criterio fue que las variables tengan una contribución al componente. El índice resume información sobre la tenencia de activos (como equipo de sonido, televisor, refrigerador, cocina a gas, entre otros), el material del piso y la disponibilidad de electricidad, agua y servicios higiénicos. Se divide a los hogares en cuartiles de riqueza, y se analizan las tendencias en los hogares en el cuartil 1 (más pobre) y en el cuartil 4 (menos pobre), utilizando regresiones no paramétricas (regresión Fan).

\section{Aspectos éticos}

En cada hogar seleccionado hubo explicación de la encuesta a realizar y se solicitó el consentimiento escrito respectivo. Además, la confidencialidad fue protegida en todo momento, separando la información personal de aquella necesaria para el análisis.

\section{RESULTADOS}

La muestra estuvo conformada por 5784 viviendas con al menos 1 niño de 1 a 24 meses, ubicados en hogares de 12 departamentos, 180 distritos y 360 centros poblados. Además, 5723 madres y 3487 hermanos; el inmediatamente mayor al niño objeto del estudio que tenga entre 3 y 18 años. Los hogares están ubicados en zonas predominantemente rurales, con tasas de pobreza mayores de $50 \%$ y tasas de desnutrición de al menos $30 \%$.

La tabla 1 presenta los resultados generales del ASQ-3 para los niños de la muestra, desagregados entre los puntajes de los 6 ítems de la escala original (puntajes entre 0 y 60), los puntajes de las 3 preguntas adicionales (puntajes entre 0 y 30) y los puntajes del agregado de los 9 ítems (puntajes entre 0 y 90). 
Tabla 1. Resultados del ASQ según escalas evaluadas y total

\begin{tabular}{|c|c|c|c|c|c|}
\hline ESCALAS & $\begin{array}{c}\text { NÚMERO DE } \\
\text { OBSERVACIONES }\end{array}$ & $\begin{array}{l}\text { PUNTAJE } \\
\text { PROMEDIO }\end{array}$ & $\begin{array}{l}\text { DESVIACIÓN } \\
\text { ESTÁNDAR }\end{array}$ & \multicolumn{2}{|c|}{$95 \% \mathrm{CI}$} \\
\hline \multicolumn{6}{|c|}{ Puntajes sobre el total de ítems regulares (máximo 60) } \\
\hline Comunicación & 5.858 & 40,85 & 13,55 & 40,51 & 41,20 \\
\hline Motora gruesa & 5.832 & 41,14 & 14,00 & 40,78 & 41,50 \\
\hline Motora fina & 5.854 & 42,39 & 12,32 & 42,08 & 42,71 \\
\hline Res. Problemas & 5.848 & 43,41 & 12,58 & 43,09 & 43,76 \\
\hline Socio-Individual & 5.855 & 43,33 & 11,49 & 43,04 & 43,63 \\
\hline \multicolumn{6}{|c|}{ Puntajes sobre el total de ítems adicionales (máximo 30) } \\
\hline Comunicación & 815 & 20,15 & 9,02 & 19,53 & 20,77 \\
\hline Motora gruesa & 888 & 16,06 & 9,70 & 15,43 & 16,70 \\
\hline Motora fina & 837 & 16,09 & 9,55 & 15,45 & 16,74 \\
\hline Res. Problemas & 950 & 18,72 & 8,89 & 18,16 & 19,29 \\
\hline Socio-Individual & 786 & 18,29 & 9,28 & 17,64 & 18,94 \\
\hline \multicolumn{6}{|c|}{ Puntajes sobre el total de ítems adicionales (máximo 90) } \\
\hline Comunicación & 5.858 & 43,66 & 18,69 & 43,18 & 44,14 \\
\hline Motora gruesa & 5.832 & 43,58 & 18,30 & 43,11 & 44,05 \\
\hline Motora fina & 5.854 & 44,70 & 16,63 & 44,27 & 45,12 \\
\hline Res. Problemas & 5.848 & 46,45 & 17,77 & 45,99 & 46,90 \\
\hline Socio-Individual & 5.855 & 45,79 & 16,07 & 45,38 & 46,20 \\
\hline
\end{tabular}

Fuente: Base de datos del documento línea de base para la evaluación de impacto del Programa Cuna Más - SAF. Elaboración: Propia.

A continuación, se emplean los puntos de cortes establecidos por los autores para determinar la presencia de riesgo en el retraso en el desarrollo de los niños. La información que se presenta se sustenta en aproximaciones tomando como referencia los puntos de corte según a las normas del manual ASQ-3. 
Tabla 2. Resultados del ASQ por sexo del niño según escalas evaluadas

\begin{tabular}{cccc}
\hline ESCALAS & NORMAL & MONITOREO & REZAGO \\
\hline Comunicación & 4.122 & 1.225 & \\
\hline Hombres & $68 \%$ & $22 \%$ & 512 \\
Mujeres & $72 \%$ & $20 \%$ & $9 \%$ \\
Motora gruesa & 3.173 & 1.223 & $8 \%$ \\
\hline Hombres & $55 \%$ & $21 \%$ & 1.463 \\
\hline Mujeres & $53 \%$ & $21 \%$ & $25 \%$ \\
Motora fina & 3.272 & 1.312 & $25 \%$ \\
\hline Hombres & $55 \%$ & $23 \%$ & 1.275 \\
Mujeres & $57 \%$ & $22 \%$ & $22 \%$ \\
Res. Problema & 4.090 & 1.041 & $22 \%$ \\
\hline Hombres & $70 \%$ & $17 \%$ & 728 \\
Mujeres & $69 \%$ & $18 \%$ & $13 \%$ \\
Socio-Individual & 3.817 & 1.284 & $12 \%$ \\
Hombres & $64 \%$ & $23 \%$ & 758 \\
Mujeres & $67 \%$ & $21 \%$ & $14 \%$ \\
\hline
\end{tabular}

Fuente: Base de datos del documento línea de base para la evaluación de impacto del programa Cuna Más - SAF. Elaboración: Propia.

En la siguiente tabla se presenta el análisis de regresión lineal buscando conocer la relación entre los resultados de las dimensiones del ASQ y las variables edad del niño, riqueza del hogar y educación de la madre.

Tabla 3. ASQ - Análisis de regresión lineal para edad, riqueza y educación de la madre ${ }^{6}$

\begin{tabular}{lccccc}
\hline & COMUNICACIÓN & $\begin{array}{c}\text { MOTORA } \\
\text { GRUESA }\end{array}$ & $\begin{array}{c}\text { MOTORA } \\
\text { FINA }\end{array}$ & $\begin{array}{c}\text { RES- } \\
\text { PROBLEMA }\end{array}$ & $\begin{array}{c}\text { SOCIO- } \\
\text { INDIVIDUAL }\end{array}$ \\
\hline & Puntajes sobre el total de ítems regulares (máximo 60) & \\
\hline $\begin{array}{l}\text { Edad en meses } \\
\text { al cuadrado }\end{array}$ & $-0,027$ & 0,013 & $-0,011$ & 0,004 & $-0,002$ \\
\hline $\begin{array}{l}\text { Índice de } \\
\text { riqueza }\end{array}$ & 0,909 & 1,158 & 1,060 & 1,059 & 1,350 \\
\hline $\begin{array}{l}\text { Años de } \\
\text { educación de la } \\
\text { madre }\end{array}$ & 0,381 & 0,297 & 0,298 & 0,238 & 0,271 \\
$\begin{array}{l}\text { cons } \\
\text { (Constante) }\end{array}$ & 43,837 & 36,718 & 42,582 & 40,886 & 41,978 \\
\hline $\begin{array}{l}\text { R-cuadrado } \\
\begin{array}{l}\text { R-cuadrado } \\
\text { ajustado }\end{array}\end{array}$ & 0,145 & 0,044 & 0,048 & 0,022 & 0,035 \\
\hline
\end{tabular}

6 El factor de expansión utilizado es "fact_cap_500_600_600a_900" relacionado con el módulo del ASQ (módulo 600). 
En general, se puede afirmar que el poder explicativo de los modelos es limitado, en la medida en que los R-cuadrados son bajos. Además, de las 5 dimensiones analizadas, la de comunicación es la que se explica mejor.

En un segundo análisis se presentan las diferencias en los puntajes de ASQ (sobre los 9 ítems) según el nivel socioeconómico del hogar ${ }^{7}$ (ver Figura 1).

Figura 1. ASQ - Gradientes socioeconómicos por nivel de riqueza, toda la muestra

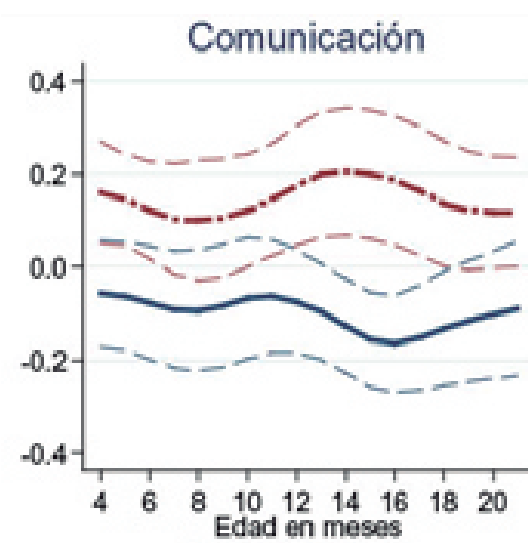

Res. Problemas

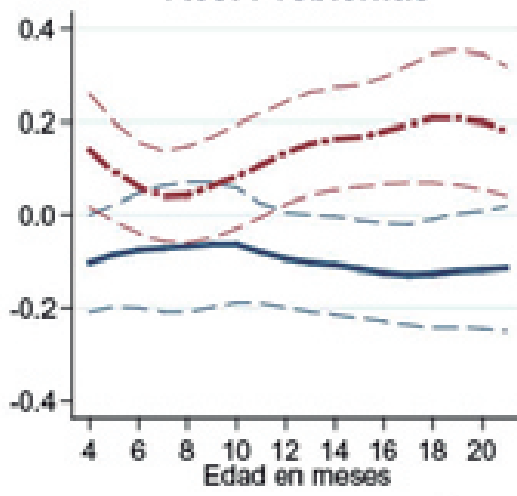

Motora Gruesa
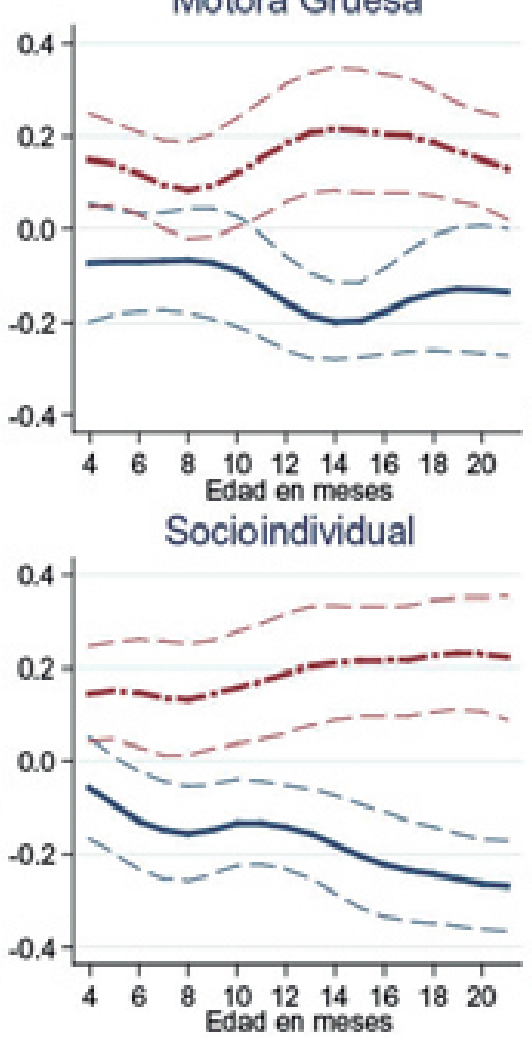

Motora Fina

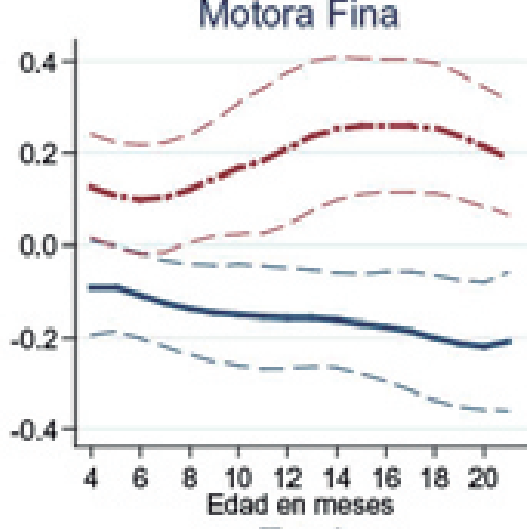

Total

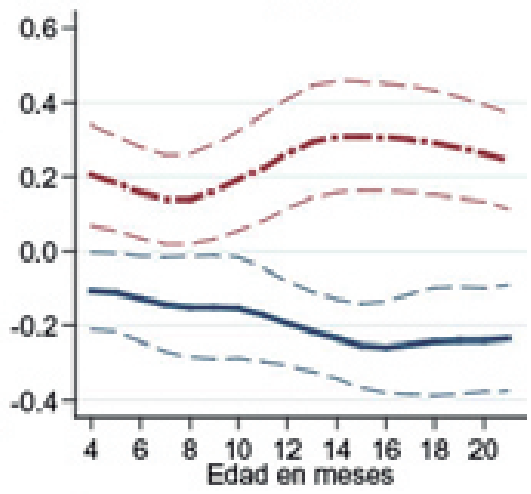

Cuartil 1

Cuartil 4

Nota. Las líneas punteadas corresponden a los intervalos de confianza.

Como se observa ${ }^{8}$, hay un gradiente socioeconómico en las 5 escalas: en promedio, los niños del cuartil 4 obtienen puntajes mayores que los niños del cuartil 1 en $0.245 \mathrm{DE}$ para resolución de problemas y comunicación, $0.27 \mathrm{DE}$ en motora

7 Para esto se construyó un índice de riqueza utilizando el método de componentes principales. El índice resume información sobre la tenencia de activos (como equipo de sonido, televisor, refrigerador, cocina a gas, entre otros), el material del piso y la disponibilidad de electricidad, agua y servicios higiénicos. Se divide a los hogares en cuartiles de riqueza y se comparan los hogares en el cuartil 1 (más pobre) y en el cuartil 4 (menos pobre) utilizando regresiones no paramétricas (regresión Fan).

8 Todos los gráficos presentados incluyen el total de observaciones con información completa de la variable dependiente. No obstante, las gráficas de gradientes no cubren los valores en las colas de la distribución de edad, dado que la estimación en estos puntos es menos precisa por dos motivos: (i) hay un menor número de observaciones en los extremos, y (ii) los métodos no paramétricos son poco confiables en los extremos, ya que, por construcción, no existe información disponible a un lado de la ventana en torno al extremo para estimar la distribución condicionada de la variable dependiente. 
gruesa, 0.34DE en motora fina y socioindividual. Si bien entre los 4 y 8 meses la diferencia de puntajes entre los niños de los dos cuartiles es significativa solo para la escala motora gruesa y la escala socioindividual, a partir de los 10-12 meses las diferencias se vuelven significativas para todas las escalas. Los mayores gradientes se registran alrededor de los 16 meses. Los niños de 16 meses del cuartil 1 obtienen en promedio un puntaje $0.45 \mathrm{DE}, 0.67 \mathrm{DE}$ y $0.78 \mathrm{DE}$ menor que los niños del cuartil 5 en las escalas de motora gruesa, comunicación y motora fina, respectivamente. A los 18 meses la diferencia entre los niños de los dos cuartiles en el área socioindividual es de $0.83 \mathrm{DE}$ en promedio. En el caso de resolución de problemas, la diferencia es de $0.53 \mathrm{DE}$. La existencia de gradientes a tan temprana edad y su tendencia creciente (consistente con la evidencia internacional) es sugerente de fuertes brechas en desarrollo en la adolescencia y la edad adulta.

En un tercer análisis, se presentan las diferencias en los puntajes de ASQ (sobre los 9 ítems) según el nivel de educación alcanzado por la madre o cuidador principal (ver Figura 2). Se compara a niños con madres con primaria incompleta o menos, con otros cuyas madres completaron la secundaria o alcanzaron una educación superior.

Figura 2. ASQ - Gradientes socioeconómicos por educación materna
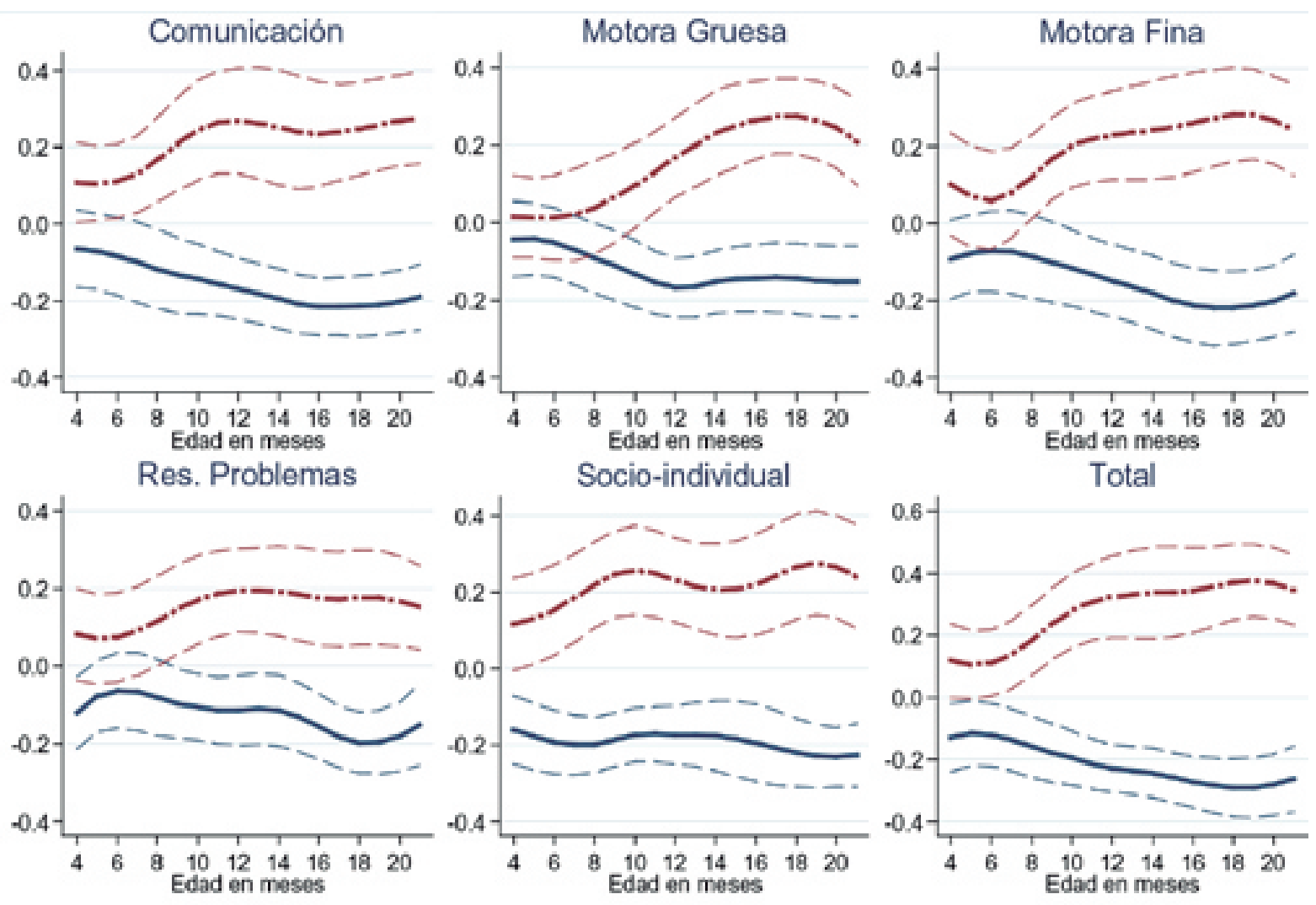

Total

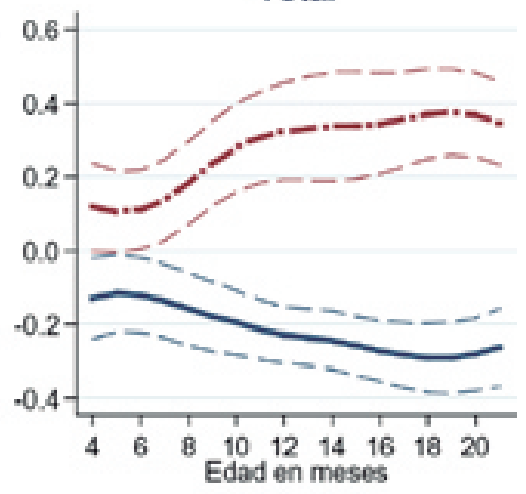

Primaria incompleta o menos

Secundaria completa o superior

Nota.- Las líneas punteadas corresponden a los intervalos de confianza. 
En las seis escalas se registran diferencias significativas entre los puntajes alcanzados por los niños con madres más y menos educadas que se intensifican con la edad. En la escala de comunicación, la diferencia de puntaje promedio para todos los niños es $0.36 \mathrm{DE}$; en las escalas resolución de problemas y socioindividual, las diferencias son de $0.27 \mathrm{DE}$ y $0.39 \mathrm{DE}$, respectivamente. Estas diferencias superan las observadas entre los hogares del cuartil 1 y 4 (0.24DE, $0.25 \mathrm{DE}$ y $0.34 \mathrm{DE}$, respectivamente). En las escalas motora gruesa y motora fina, los niños con madres más educadas obtienen puntajes $0.26 \mathrm{DE}$ y $0.33 \mathrm{DE}$ mayores que los niños con madres con menor nivel educativo, respectivamente, diferencias levemente menores que las observadas al comparar los cuartiles del ingreso $(0.28 \mathrm{DE}$ y 0.34DE, respectivamente).

Para profundizar en el análisis, se hizo un test de medias para indagar por las diferencias alcanzadas en los niveles de desarrollo infantil en cada grupo de edad, según cuartiles de riqueza y nivel educativo de la madre o responsable.

En todos los casos, las diferencias observadas en los niveles de desarrollo infantil -medidos en forma general y también en las dimensiones de comunicación, motora fina, motora gruesa, resolución de problemas y socioindividual- son estadísticamente significativas entre los grupos conformados, sea por nivel socioeconómico o por el nivel de educación de la madre.

\section{DISCUSIÓN}

En el presente artículo, se han presentado los resultados del estudio de línea de base del Servicio de Acompañamiento a Familias (SAF) del PNCM, poniendo énfasis en la revisión de los niveles de desarrollo en niños residentes en zonas rurales selectas y en la exploración de relaciones entre estos resultados y dos variables de comparación sugeridas por la literatura revisada: el índice de riqueza de las familias y la educación de la madre. En total, han participado 5859 niños y niñas con edades entre 1 y 24 meses al momento de la entrevista y 5723 madres o cuidadores principales.

Los resultados alcanzados en las cinco escalas y el puntaje total de la prueba ASQ muestran comportamientos diferentes según la edad: los puntajes de las escalas de comunicación y motora fina presentaron una tendencia decreciente con la edad, mientras que motora gruesa y resolución de problemas registran una tendencia creciente, en tanto que el puntaje total y la escala socioemocional mantienen una tendencia neutra. En los resultados puede observarse que las gradientes de las cinco escalas y el puntaje total no muestran mayores diferencias en su pendiente al acercarse al valor de 20 meses, por lo cual no se observarían diferencias como las que han sido reportadas en un estudio previo (Duckworth, 2006).

Con respecto a la estimación de diferencias según índice de riqueza, se ha encontrado que existen gradientes socioeconómicos en las cinco escalas medidas. En general, los niños del cuartil más rico en la muestra obtienen puntajes mayores que los niños del cuartil menos rico. Si bien entre los 4 y 8 meses la diferen- 
cia de puntajes entre los niños de los dos cuartiles es significativa solo para la escala motora gruesa y la escala socioindividual, a partir de los 10-12 meses las diferencias se vuelven significativas para todas las escalas. Este hallazgo guarda consistencia con lo reportado previamente, específicamente en el área de comunicación (Duckworth, 2006). Asimismo, concuerda con el reporte de Alegría Majluf, realizado con infantes de nivel socioeconómico (NSE) medio y bajo, quien halló que a los ocho meses los niveles de desarrollo entre ambos grupos eran equivalentes, pero que en los grupos de 14 y 20 meses las diferencias en puntajes a favor del grupo de NSE Medio eran crecientes (Majluf, 1983). Asimismo concuerda con un estudio multicéntrico realizado en Perú, India, Indonesia y Senegal (Fernald, 2012) y con el reporte de Kyerematen y otros (2012), realizado con 129 niños, cuya edad en promedio fue de 22 meses, de un barrio periurbano de Lima metropolitana, empleando el ASQ, donde se halló que los puntajes de tamizaje (es decir, de un posible retardo en el desarrollo) fueron significativamente mayores en la escala de comunicación en niños con menor puntaje en una escala de riqueza ad-hoc.

La existencia de gradientes que se intensifican con la edad es consistente con la evidencia internacional y sugieren fuertes brechas en desarrollo en la adolescencia y la edad adulta (Grantham-McGregor y cols, 2007; Schady, 2006; Leseman, 2002; Heckman, 2000). Las diferencias en los puntajes de desarrollo infantil encontradas en el presente estudio son similares a las reportadas en un estudio multicéntrico previamente citado (Fernald, 2012).

Los gradientes por educación materna podrían ser resultado no solo de un gradiente real, sino también podrían reflejar las diferentes habilidades de comprensión de la prueba por madres más y menos educadas. Por ejemplo, las madres con menor nivel educativo podrían responder "no, no hace" cierto ítem con mayor frecuencia porque no entienden la pregunta o porque no prestan tanta atención a los progresos de su hijo. Por definición, el gradiente en ítems por observación no está afectado por la capacidad de reporte de la madre (que puede estar correlacionada con su nivel educativo), dado que los ítems son administrados por la evaluadora. Si efectivamente madres con diferente nivel educativo tienen diferentes capacidades en la observación y reporte de las habilidades de sus hijos, se esperaría observar diferencias en los gradientes estimados por educación de la madre para ítems evaluados por observación o administración e ítems evaluados por reporte materno. Para analizar esta hipótesis, se consideran los módulos en los cuales el número de ítems que se responde por observación es similar (entre un 30 y 60\%) al número de ítems que se responden por reporte. Al compararse los gradientes por educación materna en cada grupo de ítems, se observó que las diferencias de puntajes entre los niños con madres de diferente nivel educativo se mantienen para los dos grupos de ítems. En particular, no se observa un patrón consistente que indique mayores diferencias entre los ítems que se evalúan por reporte materno en relación con los que son evaluados por observación.

Con respecto al uso del ASQ-3 como instrumento para medir el desarrollo infantil, 
este presentó muchas ventajas para los fines de esta evaluación (bajo costo, cuenta con una versión en español, los cuestionarios son de libre reproducción), pero su mayor ventaja fue la facilidad de su aplicación, característica idónea en el contexto de un estudio a gran escala que requiere la aplicación de la prueba a madres y niños de zonas rurales con bajos niveles educativos. Esta ha sido probablemente la razón por la cual la prueba ha sido usada en estudios similares (Kyerematen y cols, 2014; Fernald, 2012; Galiani, 2010). La desventaja del ASQ es básicamente la ausencia de normas a nivel nacional, por lo cual las interpretaciones que se desprenden de la categorización de los puntajes como "desarrollo típico", "monitoreo" y "posible retardo en el desarrollo" deben ser tomadas solo referencialmente.

\section{REFERENCIAS}

Attanasio, O.; Grantham-McGregor, S.M.; Fernández, C.; Fitzsimons, E.; RubioCodina, M. \& Meghir, C. (2013). Enriching the home environment of lowincome families in Colombia: A strategy to promote child development at scale. Early Childhood Matters. Bernard Van Leer Foundation.

Barnett, W. (1995). Long-term effects of early childhood programs on cognitive and school outcomes. Future of Children, 5(3), 25-50.

Duckworth, F. (2006). Development in the early years: its importance for school performance and adult outcomes. Research Report $\mathrm{N}^{\circ} 20$, Wider Benefits of Learning.

Fernald, L. K. (2012). Socioeconomic gradients in child development in very young children: Evidence from India, Indonesia, Peru and Senegal. Proceedings of the National Academy of Science (PNAS) Oct 16; 1(109), 17273-80.

Galiani, S. (2010). Scaling up Hand Washing Behavior: Findings from the Impact Evaluation Baseline Survey in Peru. Water and Sanitation Program, Technical Paper.

Gollenberg, A. L., Lynch, C. D., Jackson, L., \& McGuinness, B. M. (2009). Concurrent validity of the parent-completed Ages and Stages Questionnaires, 2nd Ed. with the Bayley Scales of Infant Development II in a low-risk sample. Child: care, health and development, 36(4), 485-490.

Grantham-McGregor, S.; Cheung, Y. B.; Cueto, S.; Glewwe, P.; Richter, L.; Strupp, B. y otros (2007). Developmental potential in the first 5 years in developing countries. Lancet (369), 60-70.

Handal, A. J., Lozoff, B., \& Breilh, J. \& Harlow, S. D. (2007a). Effect of Community of Residence on Neurobehavioral Development in Infants and Young Children in a Flower-Growing Region of Ecuador. Environmental Health Perspectives, Vol. 115, No. 1, pp. 128-133. 
Handal, A. J., Lozoff, B., \& Breilh, J. \& Harlow, S. D. (2007b). Neurobehavioral Development in Children With Potential Exposure to Pesticides. Epidemiology(18), 312-320.

Handal, A. J.; Harlow, S. D.; Breilh, J. \& Lozoff, B. (2008). Occupational Exposure to Pesticides During Pregnancy and Neurobehavioral Development of Infants and Toddlers. Epidemiology, 19(6), 851-859.

Heckman, J. (2000). Policies to foster human capital. Research in Economics, 54(1), 3-56.

Jukes, M. (2007). Impact of early childhood health and nutrition on access to education in developing countries. Paediatrics and Child Health, 17(12), 485-491.

Kyerematen, V., Hamb, A., Oberhelman, R. A., Cabrera, L., Bernabe-Ortiz, A., \& Berry, S. J. (2014). Exploratory application of the Ages and Stages (ASQ) child development screening test in a low income Peruvian shantytown population. BMJ Open. doi:10.1136/bmjopen-2013-004132

Leseman, P. (2002). Early childhood education and care for children for low income or minority backgrounds. OECD.

Limbos, M. M. \& Joyce, D. P. (2011). Comparison of the ASQ and PEDS in Screening for Developmental Delay in Children Presenting for Primary Care. Journal of Developmental \& Behavioral Pediatrics, 32(7).

Majluf, A. (1983). Desarrollo mental, postural y somático de infantes de 8, 14 y 20 meses de edad, de clase socio-económica media y baja de Lima. Revista Latinoamericana de Psicología, vol. 15, núm. 3, pp. 369-386.

Ministerio de Educación (Minedu). Resultados de la Evaluación Censal de Estudiantes 2014. Presentación web en: http://umc.minedu.gob.pe/wp-content/ uploads/2015/02/ECE-2014-Web-270215-27febv2.pdf

Pollit, E. (1984). La nutrición y el rendimiento escolar. París: Unesco.

Schady, N. (2006). Early childhood development in Latin America and the Caribbean. World Bank Policy Research Working Paper 3869.

Squires, J. B. (2009). Ages \& Stages English Questionnaires, Third Edition (ASQ3): A parent completed, child monitoring system. Baltimore: Paul H. Brookes Publishing Co. 